\title{
The effect of Teucrium polium on the duration of menstrual bleeding: A triple-blind placebo-controlled clinical trial
}

\author{
Abadian Khadige ${ }^{1}$, Zohre Keshavarz ${ }^{2}$, Faraz Mojab ${ }^{3}$, Hamid Alavi Majd ${ }^{4}$
}

${ }^{1}$ Student's Research Office, School of Nursing and Midwifery, International Branch Shahid Beheshti University of Medical Sciences, Tehran, Iran

${ }^{2}$ Assistant Professor, School of Nursing and Midwifery, Shahid Beheshti University of Medical Sciences, Tehran, Iran

${ }^{3}$ Associate Professor, Department of Pharmacogenosy, School of Pharmacology, Shahid Beheshti University of Medical Sciences, Tehran, Iran

${ }^{4}$ Associate Professor, Department of Biostatistics, School of Paramedicine, Shahid Beheshti University of Medical Sciences, Tehran, Iran

\section{Type of article: Original}

\begin{abstract}
Background: Teucrium polium (T. polium; locally called 'kalpooreh') is a herbal medicine with therapeutic effects against diabetes and fungal infections, and it has analgesic and anti-inflammatory effects as well. It is also used traditionally as a remedy in treating headache, dysmenorrhea, convulsions and gastrointestinal disorders. Moreover, its hypoglycemic, hypolipidemic and antibacterial effects have been reported in recent studies.

Objective: To compare the effectiveness of $T$. polium on reduction of menstrual bleeding.

Methods: This triple-blind, clinical trial study was performed on 70 single female students between 20 and 30 years old, studying at Shahid Beheshti University of Medical Sciences. They were allocated randomly into two groups: In the T. polium group $(\mathrm{n}=35)$, they received $250 \mathrm{mg}$ of $\mathrm{T}$. polium powder compared to 35 subjects who received placebo. For the first 3 days of menstruation, subjects were asked to take the capsules they had been given, four times daily for two consecutive menstrual cycles. Duration of menstrual bleeding was determined by a Higham chart. Data were analyzed by IBM-SPSS version 20, using repeated measures and Mann-Whitney U test.

Results: There were no differences between the two groups at baseline. Comprising the Higham chart showed that: the participants in the T. polium group had lower significant duration and amount of menstrual bleeding in the 1 st and the 2 nd menstruation cycles after treatment $(\mathrm{p}<0.05)$.

Conclusion: Teucrium polium, when taken during the first 3 days of menstruation, led to a significantly greater decrease in duration of menstrual bleeding compared to placebo.

Trail registration: The trial was registered at the Thailand Registry of Clinical Trials with the ID TCTR20160818002.

Funding: This research received financial support from Shahid Beheshti University of Medical Sciences.

Keywords: Menstrual cycle, Bleeding, Teucrium polium, Clinical trial
\end{abstract}

\section{Introduction}

Uterine bleeding is a normal and physiological process, and young women around the world have this experience in their reproductive age (1). The menstrual cycle depends on actions of the hypothalamus, pituitary, ovary and endometrium. Normal menstruation is periodically caused by decreased estradiol and progesterone production by the corpus luteum regression occurred (2). Sudden disruption of the sex steroid induces severe spasms in arterial of endometrium, and endometrial ischemia ultimately. At the same time, lysosomes degradation and proteolytic

\section{Corresponding author:}

Assistant Professor Dr. Zohre Keshavarz, School of Nursing and Midwifery, Shahid Beheshti University of Medical Sciences, Tehran, Iran. Tel.:+98.9123907712, Email: keshavarzzohre@yahoo.com Received: May 08, 2016, Accepted: August 24, 2016, Published: September 2017 iThenticate screening: August 18, 2016, English editing: September 02, 2017, Quality control: September 12, 2017 (C) 2017 The Authors. This is an open access article under the terms of the Creative Commons Attribution-NonCommercialNoDerivs License, which permits use and distribution in any medium, provided the original work is properly cited, the use is non-commercial and no modifications or adaptations are made. 
enzymes are released, which in turn, exacerbates the local destruction of tissue, and then this layer is stave (1). Most women (90\%) have menstrual cycles every 24 to 35 days. Normal bleeding of a menstrual cycle is 4-6 days. But for many women, this time varies from 2 days to 8 days long. Normal volume of menstrual bleeding is $30 \mathrm{ml}$. If the value is greater than $80 \mathrm{ml}$, it is considered unnatural (2). Iron deficiency anemia is the most common disease in women of childbearing age that causes pallor, inability to tolerate physical activity and reduction in quality of life in women within this age range (3). Some medicines can affect menstrual bleeding and reduce the severity of it, including non-steroidal anti-inflammatory drugs. But, some people have no tendency to use these chemical medications (4-7). Usage of medical plants and herbal medicine have become more popular globally in recent years than before; and this has happened due to success of this kind of medication in preventing and treating illnesses. There is a growing interest to use herbal medicine in Iran, because of the undesirable side effects of chemical medications (8). One of the herbs that have long been used medicinally is Teucrium polium (T. polium). T. polium, known locally as "Kalpooreh" grows in arid and rocky areas of Europe, the Mediterranean Basin, North Africa and South West Asia including Iran. T. polium belongs to the family of Lamiaceae. The flowers are small and have pink to white color. The oval shaped leaves are used as a traditional remedy for treating several illnesses such as stomach ache, diarrhea and convulsion (9). The plant $T$. polium is traditionally used as a remedy to treat several problems such as dysmenorrhea in Khuzestan Province, in the south west of Iran. The objective of this study was to compare the effect of T. polium and placebo in the duration of menstrual bleeding among students.

\section{Material and Methods}

\subsection{Trial design and participants}

This was a triple-blind, randomized, placebo controlled trial study, consisting of unmarried female students aged 20 to 30 years old, living in a dormitory at Shahid Beheshti University (Tehran, Iran). The study was conducted from November 21, 2014 to January 20, 2015.

\subsection{Selection criteria}

Menstrual bleeding-related variables, including age, age of menarche, age of dysmenorrhea and BMI were the same in two groups. Students who were unmarried and within age range of 20 to 30 were selected. The exclusion criteria were having a positive history of allergy to any kind of herbal medicine, usage of herbal medications during the previous 3 months, positive history of liver or kidney problems, having a history of irregular menstrual bleeding or endometriosis or vaginitis, experiencing acute stress such as grief, or missing family. During the study, there were no case reports with allergy to $T$. polium.

\subsection{Interventions}

T. polium was purchased from Barijesans Pharmaceuticals. The plant was collected from one geographic region in Kashan province, Iran. Chemical composition of the plant included: cedrol, guaiol, lindool and beta pinene (9). Then, the plant was verified and changed to powder form and put in capsules (250 mg) by the pharmacognosy laboratory in Shahid Beheshti University of Medical Sciences. The placebo capsule (250 mg starch powder) were purchased from Razac Pharmaceuticals Company (Tehran, Iran). Both capsules were the same regarding color, smell and shape. The safety dose was $250 \mathrm{mg}$ for $T$. polium based on the PDR (Herbal Medicine) book (10). T. polium and Placebo capsules were taken four times daily (For the first 3 days of menstruation). The intervention continued for two consecutive menstrual cycles. Evidence in literature revealed that the toxicity dose of the plant on kidney and liver $(11,12)$ is caused by permanent usage therefore it does not occur in alternative use of the plant $(3$ days monthly in dysmenorrhea) (13). The reliability of the Higham chart was assessed by Test-retest methods $(\mathrm{r}=0.85)$. Also, the demographic characteristics of participants such as age, body mass index (BMI), educational level, and occupation of the parents, exercise program, and stressful factors in the past 6 months were collected. The subjects were allowed to take NSAIDs if required. However, they were asked to take these medications $\geqslant 1$ hour after taking the given capsules by the researcher and they were excluded from the study. A self-reported checklist was used for collecting data on the number of sedative drugs taken for large amounts of blood loss. During the first three days of menstruation, the amount of blood loss and the duration of bleeding was recorded in each sample on the Higham chart daily. In this chart, samples recorded the value of their daily bloodshed, in fact, the number of pads used from 1-8 representing the consecutive days of menstrual period were recorded, and matched each illustration. Higham chart validity has been established in many studies, in which it has a wide range of applications, and is considered as one of the most useful and reliable measures for menstrual bleeding $(14,15)$. 


\subsection{Sample size and statistical analysis}

The sample size was calculated to be 70 subjects. This sample size was calculated based on the results of previous studies $(5,6)$ by assuming the test power of $80 \%$ and a confidence level of $95 \%$. IBM@ SPSS $\odot$ Statistics version 20 (IBMC Corp., Armonk, NY, USA) was used for data analysis. Descriptive statistics, t-test, repeated measure, and The Mann-Whitney U test were used for inferential statistical analyses.

\subsection{Randomization and blinding}

Randomly, computer-generated numbers were employed to divide participants into two groups for taking T. polium $(n=35)$ or Placebo $(n=35)$. Participants, researchers and statistic advisors were kept blinded in allocating treatment.

\subsection{Research ethics}

The study protocol was approved by the Research and Ethics Committee of Shahid Beheshti University of Medical Sciences with code: SBMU2.REC.1394.151. Students were aware of the purpose and methods of the study and provided with written consent forms for participation. The authors confirm that all interventions were safe and subjects could exclude themselves from research at any stage and there was no forced obligation to participate in the study.

\section{Results}

In total, 70 female students were enrolled in this study after applying the exclusion criteria, and were divided into two groups, those who received T. polium $(250 \mathrm{mg})(\mathrm{n}=35)$ and those who received placebo $(\mathrm{n}=35)$. Results revealed that there were no differences in demographic characteristics between the two groups. The mean age of both groups was 21 years old, mean age of menarche was 13, mean duration between cycles was 26 days, and mean duration of menstruation days was 6 days for the two groups. Duration of menstrual bleeding at baseline had no significant differences between the groups ( $>00.05$ ). In the T. polium group, duration of menstrual bleeding reduced from 6.3 (1.1) at baseline to 4.5 (8.0) in the third cycle; compared to the placebo group which decreased from 5.9 (1.3) to 5.6 (1.3). Duration of menstrual bleeding was significantly differed in each intervention cycle in the T. polium group $(p<0.05)$. Finally, results revealed that: there was significant difference in duration of menstrual bleeding between the two groups $(\mathrm{p}<0.05)$. No side effect was reported in both groups of $T$. polium and placebo.

\section{Discussion}

This paper is the report of a comparative study on the effects of $T$. polium and placebo on the alleviation of duration of menstrual bleeding. T. polium known as "Kalpooreh" is used as a remedy for many purposes in traditional medicine. It has been used traditionally for treating headache, dysmenorrhea, gastrointestinal problems and convulsion. The plant possesses therapeutic effects including anti-inflammatory, anti-nociceptive, anti-diarrhea, anti-spasmodic and moreover, hypolipidemic, hypoglycemic and anti-bacterial, which have been reported in previous research (16). Results of this study revealed that usage of the plant can decrease the duration of menstrual bleeding. In two different studies Gharib Naseri et al. revealed the antispasmodic effect on uteri of virgin rats (13) and also, they recognized the anti-spasmodic effect of T. polium on the gastrointestinal system (9). A study by Allahtavakoli et al. stated that hydro-alcoholic extract of $\mathrm{T}$. polium had a reductive effect on castor oil-induced diarrhea in male rats (16). Antispasmodic effects of $T$. polium were confirmed in animal models (rat) $(13,16)$. Phytochemical studies have shown that flavons and flavonoids are two major components in T. polium extracts and cause the antispasmodic effects in T. polium (16) which may justify its effectiveness in reduction of menstrual bleeding. Our findings indicated that T. polium significantly reduces menstrual bleeding. Karimian et al. compared the matricaria chamomilla and mefenamic acid on menstrual bleeding and observed matricaria chamomilla reduced duration of bleeding like mefenamic acid (17). Mirabi et al. demonstrated that valerian had a reductive effect as $T$. polium on menstrual bleeding (18). All of these studies demonstrate that natural remedies are effective medicine in cases of treating high amounts of menstrual bleeding. The effectiveness of $T$. polium on reduction of menstrual bleeding and its harmlessness, have been observed in this study. T. polium is suitable in decreasing menstrual bleeding in subjects. Moreover, by temporary usage of the plant, no side effects were reported by the participants. For further research regarding the effects of $T$. polium on other symptoms associated with menstrual phenomena, the efficacy and safety of further amounts of this plant are needed. Based on present results, further studies are recommended to compare $T$. polium with anti-inflammatory medications.

\section{Conclusions}

The current study indicated that T. polium decreases menstrual bleeding. Adverse effects were not reported for $T$. polium, therefore, the herb can be administered in limited dosage safely to manage this condition. We recommend 
http://www.ephysician.ir

other researchers to design different clinical trials using T. polium in different issues and also measure the probable side effects.

\section{Acknowledgments:}

The authors appreciate the participants in this study. Also, we appreciate the deputy of research at the Nursing and Midwifery School of Shahid Beheshti University of Medical Sciences.

\section{Conflict of Interest:}

There is no conflict of interest to be declared.

\section{Authors' contributions:}

All authors contributed to this project and article equally. All authors read and approved the final manuscript.

\section{References:}

1) Berek JS. Berek \& Novak gynocology.15th ed. Phiadelphia: Lippncott Williams \& Wilkins; 2012: 147484.

2) Fritz MA, Speroff L. Clinical Gynecology Endocrinology and Infertility. 8th ed. Lippincott Williams \& Wilkins; 2011: 200-580.

3) Andreoli TE, Cecil RF. Cecil Essentials of medicin. 6th edithion. W.B. Saunders; 2004.

4) Pormaleki S, Najar S, Montazery S, Haghighizadeh MH. Comparison between the Effects of Zintoma (Ginger) and Mefenamic Acid on After Pain during Postpartum in Multiparous Women. IJOGI. 2013; 16(79): 18-25.

5) Ozgoli G, Goli M, Moattar F. Comparison of effects of ginger, mefenamic acid, and ibuprofen on pain in women with primary dysmenorrhea. J Altern Complement Med. 2009; 15(2): 129-32. doi: 10.1089/acm.2008.0311. PMID: 19216660.

6) Karimian Z, Sadat Z, Abedzade M, Sarafraz N, Kafae Atrian M, Bahrami M. Comparison the Effect of Mefenamic Acid and Matricaria Chamomilla on Primary Dysmenorrhea in Medical University Students. J Ardabil Univ Med Sci. 2013; 4(13): 413-20.

7) Modarres M, Mirmohamadi AM, Oshrie Z, Mehran A. Comparison of the Effect of Mefenamic Acid and Matricaria Camomilla Capsules on Primary Dysmenorrhea. Babol University Medical Scince journal. 2010; 13(3): 50-8.

8) Cunningham C, Weathington BL, Pittenger DJ. Understanding and Conducting Research in the Health Sciences. John Wiley \& Sons; 2013: 45-60.

9) Gharib Naseri M, Omidi Birghani F. Antispasmodic effect of Teucrium polium leaf extract on rat ileum. Pajohande. 2007; 12(1): 59-67.

10) Lagow B. and e. al, eds. PDR(Herbal Medicines)The Information Standard For Complementary Medicin. 3th edition. 2004, Thomson PDR at montvale, NJ07645-1742. 649-650.

11) Rafieian-Kopaei M, Nasri H, Baradaran A. Teucrium Polium: liver and kidney effects. J Red Med Sci. 2014; 19(5): 478-9. PMID: 25097635, PMCID: PMC4116584.

12) Bahramikia S, Yazdanparast R. Phytochemistry and medicinal properties of Teucrium polium L. (Lamiaceae). Phytother Res. 2012; 26(11): 1581-93. doi: 10.1002/ptr.4617. PMID: 22344867.

13) Gharib Naseri MK, Omidi BF, Vakilzade G. Spasmolytic effect of teucrium polium on virgin rat uterus. Iranian Journal of Basic Medical Sciences. 2005; 8(1): 31-6.

14) Higham JM, O'brien PM, Shaw RW. Assessment of menstrual blood loss using a pictorial chart. BJOG. 1990; (8): 734-9. PMID: 2400752.

15) Reid PC, Coker A, Coltart R. Assessment of menstrual blood loss using a pictorial chart: a validation study. BJOG. 2000; 107(3): 320-2. doi: 10.1111/j.1471-0528.2000.tb13225.x. PMID: 10740326.

16) Allahtavakoli M, Moradi R, Samsi S, Afsharmanesh K. Effect of hydro-alcoholic extract of Teucrium polium on castor oil-induced diarrhea in male rat. Zahedan Journal of Research in Medical Sciences. 2013; 15(6): 54-7.

17) Karimian K, Abedzade M. Comparison of matricaria chamomilla and mefenamic acid on menstrual bleeding. IJOGI. 2015; 18(157): 11-7.

18) Mirabi P, Dolatian M, Mojab F, Alavimajd H. Effect of valerian on systematic manifestation of menstruation. Journal of medicinal plants. 2011; 11(44): 155-63. 